\title{
Tuberculosis: A Brief Review
}

\author{
Mayra Lucy de Macedo Targinoa ${ }^{1}$, Iasmim Lima Menezesa ${ }^{1}$, Laísa Patrícia da Silva Moreira ${ }^{1}$, \\ Ernani Canuto Figueiredo Juniorb ${ }^{2}$ and Sandra A Marinhoc ${ }^{3 *}$ \\ ${ }^{1}$ Undergraduate student, Dentistry Course, State University of Paraiba - Universidade Estadual da Paraíba (UEPB), Campus \\ VIII, Brazil
}

${ }^{2}$ MSC- Professor, Dentistry Course, State University of Paraiba - Universidade Estadual da Paraíba (UEPB), Campus VIII, Brazil

${ }^{3}$ PhD- Professor, Dentistry Course, State University of Paraiba - Universidade Estadual da Paraíba (UEPB), Campus VIII, Brazil

*Corresponding author: Sandra A Marinho, Dentistry Course, UEPB, Rua Coronel Pedro Targino s/n, Centro, 58233-000, Araruna, PB, Brazil

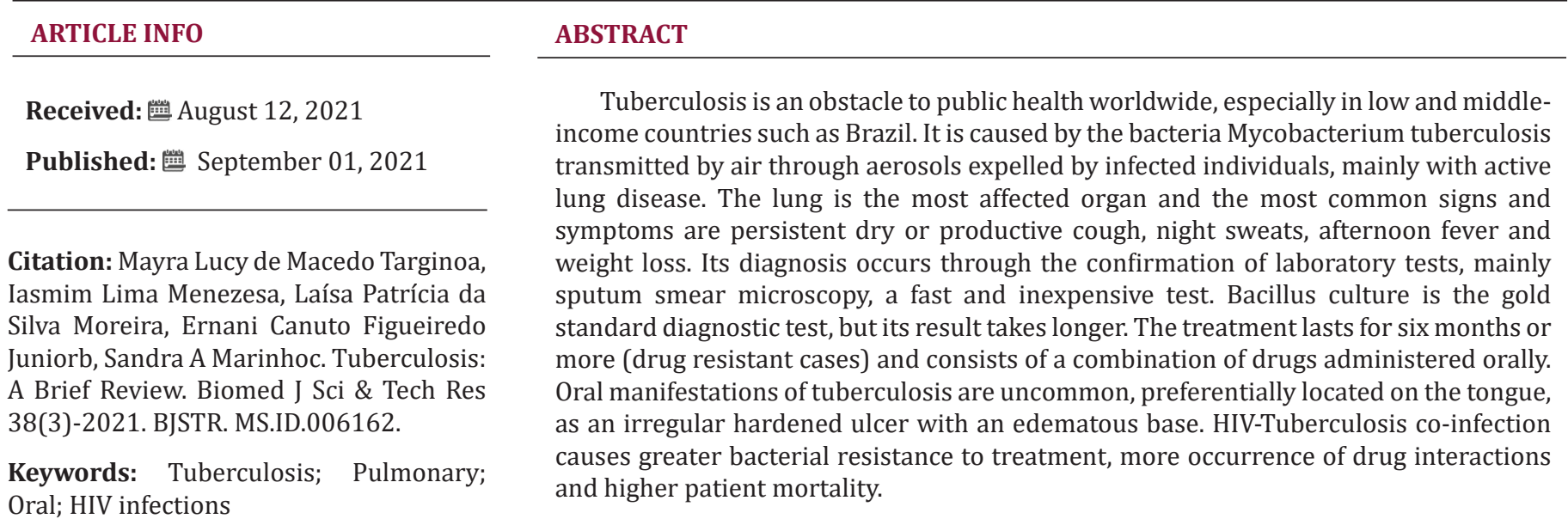

\section{Introduction and Review}

Tuberculosis (TB) is a bacterial infectious disease, caused by Mycobacterium tuberculosis, considered the most deadly infectious disease in adults, being among the top 10 causes of death in the world [1]. In 2019, it is estimated 10 million people were infected and 1.2 million died from the disease worldwide [2]. However, in the first half of 2020, there was a $25-30 \%$ decrease in the number of reported TB cases, when compared to the same period in 2019. This was one of the impacts of the Covid-19 pandemic, which led to a decrease in detection cases, the concentration of financial resources and data collection, with delay in the goals and measures to be taken [3].

TB is related to poverty, poor sanitary conditions and poor structure and effectiveness of health control programs and services in underdeveloped countries, reflecting their inequality [4,5]. In America, Brazil is one of the most affected countries by TB, being among the 30 countries with the highest burden of disease and HIV-
TB co-infection [6]. In 2018, 72,788 new cases and 4,490 deaths were recorded in Brazil, with an incidence coefficient of 34.8 cases per 100,000 inhabitants and a mortality coefficient of 2.2 deaths per 100,000 inhabitants [5,7]. In 2019, the number of new cases reached 96,000 , which $11.4 \%$ were related to HIV-TB co-infection. 6,700 deaths were recorded, with a mortality rate of 3.17 per 100,000 inhabitants [2]. M. tuberculosis affects mainly young male adults. The main form of transmission occurs through the airways, in contact with aerosols produced by the speech, sneeze or cough of contaminated people [5].

\section{Tb Types / Stages}

After the first contact with M. tuberculosis (Primary TB), it usually lodges in the lung and the infection progresses to a latent state (TB infection), or it can progress acutely (TB disease), which rarely occurs. During the latency period, patients are asymptomatic, and the bacillus is inactive in the lung. In the latency stage, only about 
10 to $15 \%$ of those infected develop the clinical signs and symptoms (TB disease) at some point in life, which usually occurs after two years from the infection. That percentage increases if the patient is immunologically compromised. In case of an immunological fail, the bacterium is reactivated and the patient shows signs and symptoms (post-primary or Secondary TB), initiating the active stage of the disease (TB disease). Most of the time, Secondary TB manifests in the lung (Pulmonary TB), but it can also affect other sites (Extrapulmonary TB). Active TB infection is the period of greatest disease transmission, as the bacterium multiplies in an unrestrained manner and the patient eliminates bacilli through sputum (bacilliferous patient), increasing the contagion $[5,8,9]$.

Pulmonary TB is the most prevalent type and affects primarily the lung, and when it affects other locations, it is called Extrapulmonary TB $[4,5]$. Primary pulmonary TB is more common in children, being less infectious and secondary pulmonary TB (post-primary) predominates in adolescents and young adults. Commonly, persistent dry or productive cough (more than two weeks), night sweats, afternoon fever (above $38^{\circ} \mathrm{C}$ ) without chills, and weight loss, are common signs and symptoms. The severe form of Pulmonary TB is called Miliary Tuberculosis and it is common in immunocompromised patients ocurring when M. tuberculosis spread acutely in the bloodstream (bacteremia) $[5,8]$.

Another serious form is Meningoencephalic TB, which occurs when bacterial spread reaches the brain, accounting for $3 \%$ of HIVnegative cases and $10 \%$ of extrapulmonary HIV-TB cases positive $[5,8]$. The extrapulmonary forms are mainly paubacillary, being less infectious. They present symptomatology according to the affected organ, being Pleural TB the most common form in nonimmunocompromised patients. Peripheral ganglionary (lymph node) TB is the most frequent form of Extrapulmonary TB in children and in HIV-positive patients. Other forms are: pericardic $\mathrm{TB}$, bone TB and tuberculous pleural empyema, which is a rupture of a tuberculous cavity into the pleural space [5].

\section{Oral Manifestations of Tb}

Oral TB is a rare type of Extrapulmonary TB, corresponding to $0.1-5 \%$ of TB infections. Oral TB can be primary (when the first infection occurs in the mouth, by direct inoculation - close contact with the infected individual who is expectorating bacilli, such as kisses, mouth-to-mouth breathing or even aerosols for dental treatment) or secondary $[10,11]$, resulting from the spread of the M. tuberculosis, usually by hematogenous or lymphatic routes, and by direct autoinoculation, in which the individual expectorates the Mycobacterium and it penetrates in favorable oral exposed areas, such as alveolus after tooth extraction and ulcerated or fissured areas of that individual $[12,13]$.
In children and adolescents, primary lesions are usually found in gums, inflammed areas or tooth extraction sites and they are associated with cervical lymphadenopathy. Secondary lesions are more common in older people and affects mainly the tongue $[10,11]$. It presents as an irregular hardened ulcer, poorly defined with a granular and swollen base [12,14,15]. Oral TB always must be considered when the lesions do not respond to therapy with conventional antibiotics and anti-inflammatory drugs [11]. However, clinical diagnosis is nonspecific and similar to other manifestations, requiring a carefully anamnesis (detailing the patient's history), and a complete physical examination, biopsy and chest radiographic exam to confirm the diagnosis $[13,14]$.

\section{Hiv-Tb Co-Infection}

HIV-TB co-infection is the leading cause of death in HIVpositives, who are 19 times more likely to develop TB [15]. Humoral immunity is compromised, impairing the action of antibodies, making HIV-positives more susceptible to the progression of TB [16]. Worldwide, the HIV-TB co-infection rate represented 208,000 of TB deaths in 2019. The co-infection rate reached $11.4 \%$ of new cases, and only $47.5 \%$ of these patients had access to antiretroviral treatment $[2,7]$. Co-treatment HIV-TB should be established, with concern for drug interaction and tolerance of the infected patient. Delayed diagnosis can lead to multidrug-resistant tuberculosis, increasing the mortality $[8,15]$. In HIV-TB patient, sputum tests and Mycobacterial culture should also be ordered. However, the molecular tests of nucleic acid amplification provide a quicker diagnosis [5,15]. Among these tests, the most widely used is GeneXpert MTB/RIF (Cepheid, USA), an automated real-time PCR system that amplifies a gene from a specific Mycobacterium sequence. It identifies the bacterium and the mutations caused by rifampicin, being recommended for the initial diagnosis of adults and children, of HIV-TB co-infection and TB resistant to rifampicin $[17,18]$. Furthermore, the HIV detection test is recommended for all diagnosed TB patients or those with negative smear tests [15].

\section{Tb Diagnosis}

Some confirmatory tests are performed to determine the diagnosis of $\mathrm{TB}$, since there is not a single test with the necessary sensitivity and specificity $[8,13]$. In latency stage, patients are asymptomatic and to identify infected individuals, the tuberculin skin test (purified protein derivative- PPD) and the serological test for gamma interferon-release are applied [8]. However, serological tests have low sensitivity and specificity [19]. A positive PPD result indicates only TB infection and it is not sufficient for the diagnosis of active disease. It consists of the intradermal application of tuberculin with reading after 72 to 96 hours. If the diameter of the hardened area is greater than $10 \mathrm{~mm}$, it indicates previous contact 
with M. tuberculosis or the individual has been vaccinated with Bacille Calmette-Guerin (BCG) in the last two years and may be sick or not [20].

Inthe activeform of the disease, themostcommon testis the direct microscopic examination, known as sputum smear microscopy, as it is an easy, fast, inexpensive and highly specific method. It uses Ziehl-Nielsen staining, which allows the identification of the acidresistant bacillus (BAAR). This method is responsible for $70-80 \%$ detection of pulmonary TB, with the collection of two sputum samples with a result within 48 hours [5,21,22]. However, it has low sensitivity in patients HIV-TB co-infected [23]. Extrapulmonary TB has a lower bacterial load, which reduces sensitivity in the sputum test. Thus, it is highly recommended to perform a biopsy to investigate all extrapulmonary forms, or even needle aspiration cytology to obtain the diagnosis [8].

Culture for Mycobacteria with antimicrobial susceptibility testing is the gold standard for TB diagnosis, with high sensitivity and specificity. The culture identifies the Mycobacterium and the sensitivity test distinguishes whether it is a tuberculous Mycobacterium. Culture is recommended for all suspected cases, regardless of the result of sputum smear microscopy and the rapid molecular tests for TB (RMT-TB). The culture result is obtained after a few weeks [5,21]. Regardless of the other tests results carried out, culture for Mycobacterium is always indicated, with an antimicrobial sensitivity test $[5,24]$. Another available method is the rapid molecular test for TB (RMT-TB), which, using the polymerase chain reaction (PCR) technique, detects the DNA of M. tuberculosis and also strains resistant to rifampicin in sputum samples. The test result is obtained in two hours, in addition to presenting greater sensitivity in sputum samples than sputum smear microscopy. It is indicated for the diagnosis of new cases of pulmonary and laryngeal TB in adults and adolescents [5].

In addition, for patients with suspected pulmonary TB, a chest $\mathrm{X}$-ray is requested to rule out another lung disease, and also to assess the extent and evolution of the case during the treatment, as well as the existence of a residual infection. A computed tomography scan of the chest may also be requested [5,24].

\section{Tb Prevention}

The prevention against the complications of TB is done through the Bacille Calmette-Guerin (BCG) vaccine, preventing meningeal and miliary tuberculosis, the most severe forms of the disease. The vaccine should preferably be applied within the first 12 hours after birth and it is contraindicated for HIV children up to five years and when they weigh less than $2 \mathrm{~kg}[5,25]$. However, BCG has variable efficacy, being more effective in prevention of children, especially newborns, not showing the same results in adults against pulmonary $\mathrm{TB}$. The vaccine is more effective in immunologically immature individuals, who have not had any contact with $\mathrm{M}$. tuberculosis, or any other Mycobacteria [5,26].

\section{Tb Treatment}

The treatment is currently guided by the supervised treatment strategy (Directly observed treatment short-course-DOTS), which follows the basic scheme of short duration (about six months), using drugs with combined fixed dose (CFD) [27]. Therapy is performed orally and combines drugs to prevent bacterial resistance, through a basic scheme that consists of two phases: intensive, lasting two months, combining rifampicin, isoniazid, pyrazinamide and ethambutol, known as the RHZE regimen. The second phase (maintenance) lasts four months and uses the rifampicin and isoniazid regimen ( $\mathrm{RH}$ regimen). The regimen depends on the patient weight and it is used for all patients above 10 years old $[5,8,27]$. Patients HIV-TB follow the same treatment regimen. However, in these patients it is more frequent adverse reactions as treatment interruption, disease recurrence and risk of drug interactions. TB treatment must be started first and depending on the immunosuppression (TCD4 lymphocyte count) of the individual, antiretroviral therapy is instituted. If the TCD4 cell count is less than $50 / \mathrm{mm} 3$ (advanced immunosuppression), therapy should start as early as the second week after the TB treatment. However, when the count is TCD $4 \geq 50 / \mathrm{mm} 3$, treatment can be performed at the end of the intensive TB treatment phase, around the eighth week [5,28].

\section{Discussion}

Young adult men are the most affected by the TB. After primary infection in the lung, the bacilli remain latent and in a patient's immune suppression, the bacterium is reactivated, appearing signs and symptoms (secondary TB) and it initiates the active stage (TB disease). It is a period of greatest transmissibility of the disease, as the bacteria begins to multiply and the patient eliminates bacilli through sputum $[5,8,9]$. The main signs and symptoms are moderate fever (in the afternoon), cough, weight loss and night sweats [20]. The most test used for the diagnosis is the direct sputum smear microscopy, as it is cheap, quick and easy test. It is responsible for the detection of 70-80\% of pulmonary TB, the most common manifestation of the disease $[5,21,22]$. Extrapulmonary TB has a lower bacterial load, which decreases the sensitivity of sputum smear microscopy. Thus, biopsy or fine needle aspiration is indicated [8]. However, culture for Mycobacteria with antimicrobial susceptibility testing is the most specific and sensitive method, being the gold standard for diagnosing TB $[5,21]$. Chest radiography should also be requested for patients with suspected pulmonary $\mathrm{TB}$, to rule out another lung disease and to assess the evolution during the treatment, as well as the existence of a residual infection $[5,25]$. The rapid molecular test for TB (TRM-TB) detects bacterial 
DNA and strains resistant to rifampicin, in sputum samples. The result is obtained in two hours, and it is more sensitive than sputum smear microscopy [5].

In order to avoid the appearance of the most severe forms of TB (meningeal and miliary tuberculosis), the BCG vaccine should be applied, preferably, in the first 12 hours of the baby's birth. In Brazil, this vaccine is recommended for children under five years old and is contraindicated for children with HIV [5,26]. BCG is more effective in individuals who have not any contact with M. tuberculosis or other mycobacterial organisms $[5,27]$. The TB treatment consists of a sixmonth regimen that uses drugs with a fixed dose (CFD) supervised on an outpatient basis. Initially, the oral administration of the RHZE scheme in an intensive phase (two months) takes place and then, in the maintenance phase (four months), the RH scheme is used, and doses adjusted according the patient's weight $[5,8,27]$. Patients HIV-TB follow the same treatment, but they have more adverse reactions, with greater chances of discontinuing treatment. In those patients, TB treatment should be started first and, depending on the degree of immunosuppression, antiretroviral therapy is instituted in the second or eighth week from the beginning of TB treatment $[5,28,29]$.

The main cause of death of HIV-positive patient is due to coinfection with TB [2]. Brazil is one of the countries with the highest burden of disease and HIV-TB co-infection [5,6]. In 2019, the number of new cases in Brazil reached 96,000, with 11.4\% of new cases related to HIV-TB co-infection and a mortality rate of 3.17 per 100,000 inhabitants [2]. Less than half of HIV-TB patients had access to antiretroviral with TB treatments [2,7]. The early diagnosis of TB in co-infected patients is important, since the delay in diagnosis can lead to multidrug-resistant TB, increasing the mortality chance $[2,8]$. If bacilloscopy and mycobacterial culture tests show false negative results [24], molecular PCR tests should provide quick diagnosis [2,5]. Among these tests, the most widely used is Xpert MTB/RIF, an automated real-time PCR system that amplifies a gene from a specific Mycobacterium sequence. In this way, it identifies the bacterium and also the mutations caused by rifampicin. This test is recommended for the initial diagnosis of HIV-TB co-infection and rifampicin-resistant TB in adults and children [18,19]. In addition, the HIV detection test is recommended for all patients with diagnosed TB or those with negative sputum smear tests [2].

\section{Final Considerations}

Tuberculosis is a worldwide public health problem, especially in developing countries as Brazil. The pulmonary form is the most common presentation of the disease. TB treatment is prolonged, requiring six months, and involves various drugs to prevent microbial resistance, mainly to the rifampicin. Once the treatment started, should not be interrupted. However, as it is daily and prolonged, many patients interrupt or abandon the treatment. In addition, HIV-TB co-infection causes greater microbial resistance, and more adverse reactions, resulting from drug interactions, and higher mortality of these patients.

\section{References}

1. (2021) Ministry of Health. Health Surveillance Secretariat. 2020c. National Tuberculosis Control Program. Brasil, p. 28.

2. (2020) World Health Organization (WHO). Global Tuberculosis Report. WHO/HTM/TB/2020. Geneve: WHO.

3. (2020) Pan American Health Organization (PAHO). Global progress in combating tuberculosis is at risk, says WHO.

4. Grange JM, Zumla A (2002) The global emergency of tuberculosis: what is the cause? J. R. Soc. Promot. Health. 122(2): 78-81.

5. (2019) Ministry of Health. Health Surveillance Secretariat. 2019. Tuberculosis Epidemiological Bulletin 2019. Brasília: Ministry of Health. Brasil, p. 18.

6. (2021) Ministry of Health. Health Surveillance Secretariat. 2020b. Manual of Recomendations for Tuberculosis Control in Brazil. 2. ed. Brasília: Ministry of Health. Brasil, pp. 366.

7. (2020) Ministry of Health. Health Surveillance Secretariat. 2020a. Tuberculosis Epidemiological Bulletin 2020. Brasília: Ministry of Health. Brasil, p. 40.

8. (2011) National Institute for Health and Clinical Excellence (NICE). National Collaborating Centre for Chronic Conditions (UK); Centre for Clinical Practice at Nice (UK). Tuberculosis: Clinical Diagnosis and Management of Tuberculosis, and Measures for Its Prevention and Control. London: NICE (UK). (NICE Clinical Guidelines, No. 117) PMID: 22720337.

9. (2021) Center for Disease Control and Prevention (CDC). 2020. Basic TB facts, latent tuberculosis infection and tuberculous disease.

10. Eng HL, Lu SY, Yang CH, Chen WJ (1996) Oral tuberculosis. Oral Surg. Oral Med. Oral Pathol. Oral Radiol Endod 81(4): 415-420.

11. Mignogna MD, Muzio LL, Flavia G, Ruoppo E, Sammartino G, et al. (2000) Oral tuberculosis: a clinical evaluation of 42 cases. Oral Dis 6(1): 25-30.

12. McAndrew PG, Adekeye EO, Ajdukiewicz AB (1976) Miliary tuberculosis presenting with multifocal oral lesions. Br Med 1(6021):1320.

13. Kakisi OK, Kechagia AS, Kakisis IK, Rafailidis PI (2010) Tuberculosis of the oral cavity: a systematic review. Eur J Oral Sci 118(2): 103-109.

14. Rout P, Modipalle V, Hedge SS, Patel N, Uppala S, et al. (2019) Prevalence of oral lesions in tuberculosis: A cross sectional study. J Family Med Prim Care 8(12): 3821-3825.

15. (2018) World Health Organization (WHO). HIV-Associated Tuberculosis. Geneve: WHO

16. Mumtaz S, Pabla R (2020) Oral tuberculosis. Br Dent J 229(9): 571.

17. Van Woudenbergh E, Irvine EB, Davies L, De Kock M, Hanekom WA, et al. (2020) HIV Is Associated with modified humoral immune responses in the setting of HIV/TB coinfection. mSphere, 5, e00104-001020.

18. Boehme CC, Nabeta P, Hillemann D, Nicol MP, Shenai S, et al. (2010) Rapid molecular detection of tuberculosis and rifampin resistance. $\mathrm{N}$ Engl J Med 363(11): 1010-1015.

19. Lawn SD, Nicol MP (2011) Xpert ${ }^{\oplus}$ MTB/RIF assay: development, evaluation and implementation of a new rapid molecular diagnostic for tuberculosis and rifampicin resistance. Future Microbiol 6(9): 10671082. 
20. Steingart KR, Flores LL, Dendukuri N, Schiller I, Laal S, et al. (2011) Commercial serological tests for the diagnosis of active pulmonary and extrapulmonary tuberculosis: an updated systematic review and metaanalysis. PLoS Med 8(8): e1001062.

21. Silva Jr JB (2004) Tuberculosis: Epidemiological Surveillance Guide. J Bras Epidemiol 30: S57-85.

22. Foulds J, O Brien R (1998) New tools for the diagnosis of tuberculosis: the perspective of developing countries. Int J Tuberc Lung Dis 2(10): 778-783.

23. Zumla A, Raviglione M, Hafner R, Von Reyn CF (2013) Tuberculosis. N Engl J Med 368: 745-755.

24. Steingart KR, Ng V, Henry M, Hopewell PC, Ramsay A, et al. (2006) Sputum processing methods to improve the sensitivity of smear microscopy for tuberculosis: a systematic review. Lancet Infect Dis 6(10): 664-674.

ISSN: 2574-1241

DOI: 10.26717/BJSTR.2021.38.006162

Sandra A Marinhoc. Biomed J Sci \& Tech Res

(c) (P) This work is licensed under Creative

Submission Link: https://biomedres.us/submit-manuscript.php
25. Bomanji JB, Gupta N, Gulati, P, Das CJ (2015) Imaging in tuberculosis. Cold Spring Harb. Perspect Med 5(6): a017814.

26. (2020) Ministry of Health. Health Surveillance Secretariat. 2014. Manual of rules and procedures for vaccination. Brasília: Ministry of Health. Brasil, pp. 178.

27. Mangtani P, Abubakar I, Ariti C, Beynon R, Pimpin L, et al. (2014) Protection by BCG vaccine against tuberculosis: a systematic review of randomized controlled trials. Clin Infect Dis 58(4): 470-480.

28. (2020) Ministry of Health. Health Surveillance Secretariat. Technical Manual for Tuberculosis Control in Brazil. 2. ed. Brasília: Ministry of Health, Brasil, p. 62.

29. (2016) Center for Disease Control and Prevention (CDC). Basic TB facts treatment of LTBI and TB for people with HIV.

$\begin{array}{ll}\text { BIOMEDICAL } & \text { Assets of Publishing with us } \\ \text { RESEARCHES } & \text { - Global archiving of articles } \\ \text { - Immediate, unrestricted online access }\end{array}$

